Supporting Information

\title{
DNAzyme-Controlled Cleavage of Dimer and Trimer Origami Tiles
}

\author{
Na Wu and Itamar Willner* \\ Institute of Chemistry, The Center for Nanoscience and Nanotechnology,
}

The Hebrew University of Jerusalem, Jerusalem 91904, Israel

Address correspondence to: willnea@vms.huji.ac.il

Tel: $+972-2-6585272$

Fax: $+972-2-6527715$ 


\begin{tabular}{|c|c|c|c|}
\hline \multicolumn{2}{|c|}{ Description } & Origamis Counted \\
\hline & Prior to cleavage & Singles & 69 \\
\hline & Dimers & $226(77 \%)$ \\
\hline $\mathrm{T}_{1}-\mathrm{T}_{2}$ & & In all & 295 \\
\hline & After interaction with $\mathrm{Pb}^{2+}$ ions & Singles & 143 \\
\hline & & Dimers & $72(33 \%)$ \\
\hline & & & 215 \\
\hline
\end{tabular}

Figure S1. The detailed analysis of the cleavage of the dimers $T_{1}-T_{2}$ with $\mathrm{Pb}^{2+}$-ions.

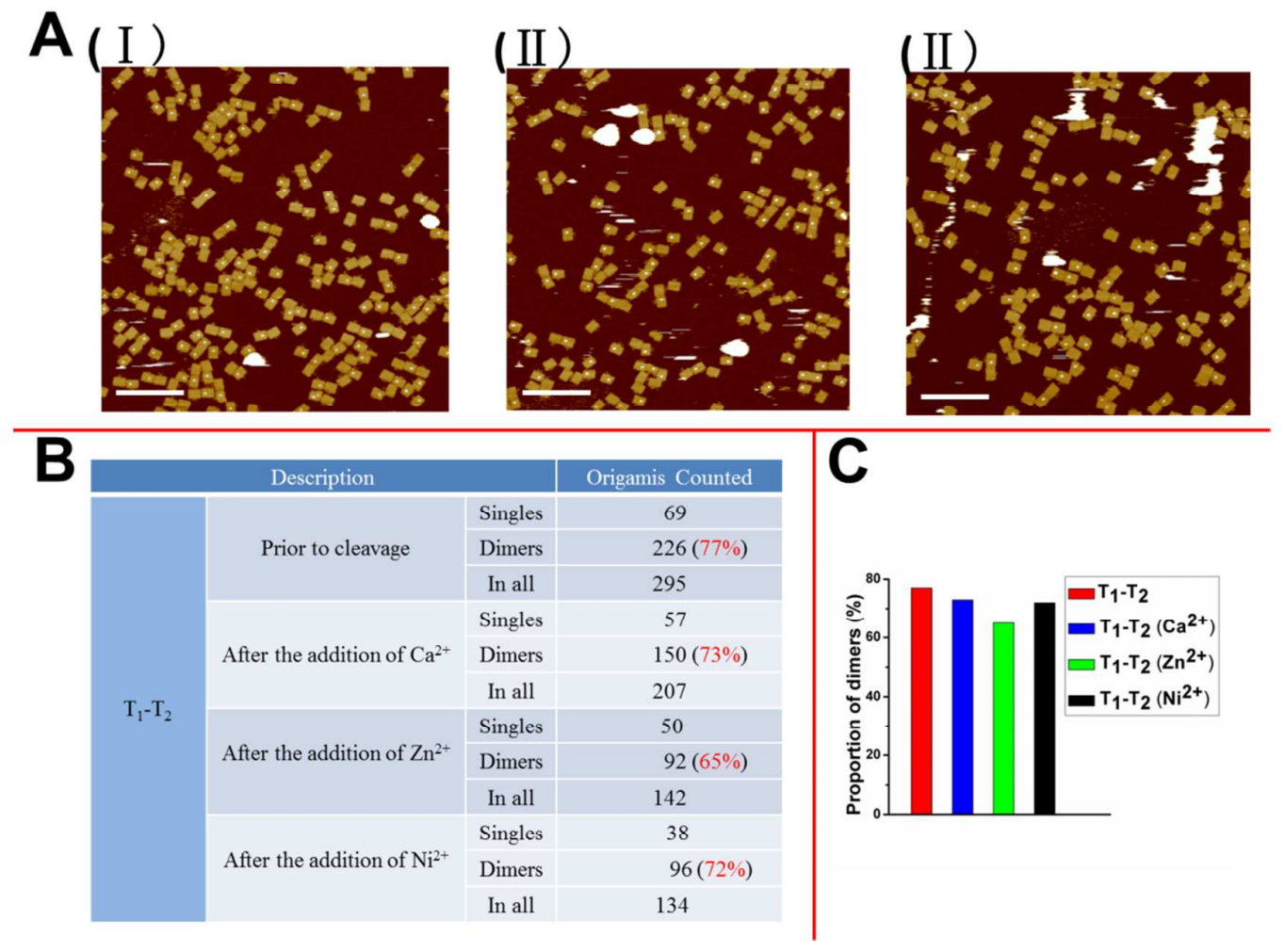


Figure S2. The selectivity of the DNAzyme to $\mathrm{Pb}^{2+}$-ions. (A) AFM images corresponding to: Panel ( I )- After the treatment of $T_{1}-T_{2}$ dimers with $\mathrm{Ca}^{2+}$-ions. Panel ( II)- After the treatment of $\mathrm{T}_{1}-\mathrm{T}_{2}$ dimers with $\mathrm{Zn}^{2+}$-ions. Panel (III)- After the treatment of $\mathrm{T}_{1}-\mathrm{T}_{2}$ dimers with $\mathrm{Ni}^{2+}$-ions. Scale bars: $500 \mathrm{~nm}$. (B) The detailed analysis of the selectivity of the $\mathrm{Pb}^{2+}$-ion-dependent DNAzyme, respectively, with $\mathrm{Ca}^{2+}$-ions, $\mathrm{Zn}^{2+}$-ions and $\mathrm{Ni}^{2+}$-ions. (C) Statistical analysis of the content of the dimer origami tiles before and after the treatments of $\mathrm{Ca}^{2+}$-ions, $\mathrm{Zn}^{2+}$-ions and $\mathrm{Ni}^{2+}$-ions respectively.

\begin{tabular}{|c|c|c|c|}
\hline & & Description & Origamis Counted \\
\hline \multirow{3}{*}{$\mathrm{T}_{3}-\mathrm{T}_{4}$} & Prior to cleavage & Singles & 66 \\
\cline { 3 - 4 } & & Dimers & $138(68 \%)$ \\
\hline \multirow{2}{*}{ After interaction with His } & In all & 204 \\
\cline { 2 - 4 } & & Singles & 252 \\
\hline & & Dimers & $36(12 \%)$ \\
\hline
\end{tabular}

Figure S3. The detailed analysis of the cleavage of the dimers $T_{3}-T_{4}$ with histidine. 


\begin{tabular}{|c|c|c|c|c|c|c|c|}
\hline \multirow[t]{2}{*}{ Description } & \multicolumn{7}{|c|}{ The number of Origami tiles / Proportion } \\
\hline & $\mathrm{T}_{1}-\mathrm{T}_{5}-\mathrm{T}_{4}$ & $\mathrm{~T}_{1}-\mathrm{T}_{5}$ & $\mathrm{~T}_{5}-\mathrm{T}_{4}$ & $\mathrm{~T}_{1}$ & $\mathrm{~T}_{5}$ & $\mathrm{~T}_{4}$ & In all \\
\hline Prior to cleavage & $120 / 46 \%$ & $44 / 17 \%$ & $20 / 7.7 \%$ & $20 / 7.7 \%$ & $22 / 8.5 \%$ & $33 / 13 \%$ & 259 \\
\hline $\begin{array}{l}\text { After interaction } \\
\text { with } \mathrm{Pb}^{2+} \text { ions }\end{array}$ & $45 / 14 \%$ & $42 / 13 \%$ & $80 / 24 \%$ & $96 / 29 \%$ & $37 / 11 \%$ & $28 / 8.5 \%$ & 328 \\
\hline $\begin{array}{c}\text { After interaction } \\
\text { with His }\end{array}$ & $15 / 7.6 \%$ & $78 / 40 \%$ & $10 / 5.1 \%$ & $27 / 14 \%$ & $25 / 13 \%$ & $42 / 22 \%$ & 197 \\
\hline $\begin{array}{c}\text { After interaction } \\
\text { with } \mathrm{Pb}^{2+} \text { ions } \\
\& \text { His }\end{array}$ & $6 / 3.6 \%$ & $14 / 8.5 \%$ & $12 / 7.3 \%$ & $49 / 30 \%$ & $45 / 27 \%$ & $39 / 24 \%$ & 165 \\
\hline
\end{tabular}

Figure S4. The detailed analysis of the cleavage of the trimers $T_{1}-T_{5}-T_{4}$ in the presence of $\mathrm{Pb}^{2+}$-ions and /or histidine.

\section{DNA Sequences}

Sequences of the staple strands for the rectangular DNA origami tiles:

$$
\begin{aligned}
& \text { CAAGCCCAATAGGAAC CCATGTACAAACAGTT } \\
& \text { AATGCCCCGTAACAGT GCCCGTATCTCCCTCA } \\
& \text { TGCCTTGACTGCCTAT TTCGGAACAGGGATAG } \\
& \text { GAGCCGCCCCACCACC GGAACCGCGACGGAAA } \\
& \text { AACCAGAGACCCTCAG AACCGCCAGGGGTCAG } \\
& \text { TTATTCATAGGGAAGG TAAATATTCATTCAGT } \\
& \text { CATAACCCGAGGCATA GTAAGAGCTTTTTAAG } \\
& \text { ATTGAGGGTAAAGGTG AATTATCAATCACCGG } \\
& \text { AAAAGTAATATCTTAC CGAAGCCCTTCCAGAG } \\
& \text { GCAATAGCGCAGATAG CCGAACAATTCAACCG } \\
& \text { CCTAATTTACGCTAAC GAGCGTCTAATCAATA } \\
& \text { TCTTACCAGCCAGTTA CAAAATAAATGAAATA } \\
& \text { ATCGGCTGCGAGCATG TAGAAACCTATCATAT }
\end{aligned}
$$


CTAATTTATCTTTCCT TATCATTCATCCTGAA GCGTTATAGAAAAAGC CTGTTTAGAAGGCCGG GCTCATTTTCGCATTA AATTTTTGAGCTTAGA AATTACTACAAATTCT TACCAGTAATCCCATC TTAAGACGTTGAAAAC ATAGCGATAACAGTAC TAGAATCCCTGAGAAG AGTCAATAGGAATCAT CTTTTACACAGATGAA TATACAGTAAACAATT TTTAACGTTCGGGAGA AACAATAATTTTCCCT CGACAACTAAGTATTA GACTTTACAATACCGA GGATTTAGCGTATTAA ATCCTTTGTTTTCAGG ACGAACCAAAACATCG CCATTAAATGGTGGTT GAACGTGGCGAGAAAG GAAGGGAACAAACTAT TAGCCCTACCAGCAGA AGATAAAAACATTTGA CGGCCTTGCTGGTAAT ATCCAGAACGAACTGA CTCAGAGCCACCACCC TCATTTTCCTATTATT CTGAAACAGGTAATAA GTTTTAACCCCTCAGA AGTGTACTTGAAAGTA TTAAGAGGCCGCCACC GCCACCACTCTTTTCA TAATCAAACCGTCACC GTTTGCCACCTCAGAG CCGCCACCGATACAGG GACTTGAGAGACAAAA GGGCGACAAGTTACCA AGCGCCAACCATTTGG GAATTAGATTATTAGC GAAGGAAAATAAGAGC AAGAAACAACAGCCAT GCCCAATACCGAGGAA ACGCAATAGGTTTACC ATTATTTAACCCAGCT ACAATTTTCAAGAACG TATTTTGCTCCCAATC CAAATAAGTGAGTTAA GGTATTAAGAACAAGA AAAATAATTAAAGCCA TAAGTCCTACCAAGTA CCGCACTCTTAGTTGC ACGCTCAAAATAAGAA TAAACACCGTGAATTT AGGCGTTACAGTAGGG CTTAATTGACAATAGA ATCAAAATCGTCGCTA TTAATTAACGGATTCG CTGTAAATCATAGGTC TGAGAGACGATAAATA CCTGATTGAAAGAAAT TGCGTAGACCCGAACG ACAGAAATCTTTGAAT ACCAAGTTCCTTGCTT TTATTAATGCCGTCAA TAGATAATCAGAGGTG AGATTAGATTTAAAAG TTTGAGTACACGTAAA AGGCGGTCATTAGTCT TTAATGCGCAATATTA GAATGGCTAGTATTAA CACCGCCTCAACTAAT CCGCCAGCCATTGCAA CAGGAAAAATATTTTT CCCTCAGAACCGCCAC CCTCAGAACTGAGACT CCTCAAGAATACATGG CTTTTGATAGAACCAC TAAGCGTCGAAGGATT AGGATTAGTACCGCCA CACCAGAGTTCGGTCA TAGCCCCCGCCAGCAA TCGGCATTCCGCCGCC AGCATTGACGTTCCAG AATCACCAAATAGAAA ATTCATATATAACGGA 


\begin{tabular}{|c|c|}
\hline 58 & TCACAATCGTAGCACC ATTACCATCGTTTTCA \\
\hline 59 & ATACCCAAGATAACCC ACAAGAATAAACGATT \\
\hline 60 & ATCAGAGAAAGAACTG GCATGATTTTATTTTG \\
\hline 61 & TTTTGTTTAAGCCTTA AATCAAGAATCGAGAA \\
\hline 62 & AGGTTTTGAACGTCAA AAATGAAAGCGCTAAT \\
\hline 63 & CAAGCAAGACGCGCCT GTTTATCAAGAATCGC \\
\hline 64 & AATGCAGACCGTTTTT ATTTTCATCTTGCGGG \\
\hline 65 & CATATTTAGAAATACC GACCGTGTTACCTTTT \\
\hline 66 & AATGGTTTACAACGCC AACATGTAGTTCAGCT \\
\hline 67 & TAACCTCCATATGTGA GTGAATAAACAAAATC \\
\hline 68 & AAATCAATGGCTTAGG TTGGGTTACTAAATTT \\
\hline 69 & GCGCAGAGATATCAAA ATTATTTGACATTATC \\
\hline 70 & AACCTACCGCGAATTA TTCATTTCCAGTACAT \\
\hline 71 & ATTTTGCGTCTTTAGG AGCACTAAGCAACAGT \\
\hline 72 & CTAAAATAGAACAAAG AAACCACCAGGGTTAG \\
\hline 73 & GCCACGCTATACGTGG CACAGACAACGCTCAT \\
\hline 74 & GCGTAAGAGAGAGCCA GCAGCAAAAAGGTTAT \\
\hline 75 & GGAAATACCTACATTT TGACGCTCACCTGAAA \\
\hline 76 & TATCACCGTACTCAGG AGGTTTAGCGGGGTTT \\
\hline 77 & TGCTCAGTCAGTCTCT GAATTTACCAGGAGGT \\
\hline 78 & GGAAAGCGACCAGGCG GATAAGTGAATAGGTG \\
\hline 79 & TGAGGCAGGCGTCAGA CTGTAGCGTAGCAAGG \\
\hline 80 & TGCCTTTAGTCAGACG ATTGGCCTGCCAGAAT \\
\hline 81 & CCGGAAACACACCACG GAATAAGTAAGACTCC \\
\hline 82 & ACGCAAAGGTCACCAA TGAAACCAATCAAGTT \\
\hline 83 & TTATTACGGTCAGAGG GTAATTGAATAGCAGC \\
\hline 84 & TGAACAAACAGTATGT TAGCAAACTAAAAGAA \\
\hline 85 & CTTTACAGTTAGCGAA CCTCCCGACGTAGGAA \\
\hline 86 & GAGGCGTTAGAGAATA ACATAAAAGAACACCC \\
\hline 87 & TCATTACCCGACAATA AACAACATATTTAGGC \\
\hline 88 & CCAGACGAGCGCCCAA TAGCAAGCAAGAACGC \\
\hline 89 & AGAGGCATAATTTCAT CTTCTGACTATAACTA \\
\hline 90 & TTTTAGTTTTTCGAGC CAGTAATAAATTCTGT \\
\hline 91 & TATGTAAACCTTTTTT AATGGAAAAATTACCT \\
\hline 92 & TTGAATTATGCTGATG CAAATCCACAAATATA \\
\hline 93 & GAGCAAAAACTTCTGA ATAATGGAAGAAGGAG \\
\hline 94 & TGGATTATGAAGATGA TGAAACAAAATTTCAT \\
\hline 95 & CGGAATTATTGAAAGG AATTGAGGTGAAAAAT \\
\hline 96 & ATCAACAGTCATCATA TTCCTGATTGATTGTT \\
\hline 97 & CTAAAGCAAGATAGAA CCCTTCTGAATCGTCT \\
\hline 98 & GCCAACAGTCACCTTG CTGAACCTGTTGGCAA \\
\hline 99 & GAAATGGATTATTTAC ATTGGCAGACATTCTG \\
\hline 100 & TTTT TATAAGTA TAGCCCGGCCGTCGAGAGGGTTGA \\
\hline 101 & TTTT ATAAATCC TCATTAAATGATATTCACAAACAA \\
\hline
\end{tabular}




\begin{tabular}{|c|c|}
\hline 102 & TTTT AATCAGTA GCGACAGATCGATAGCAGCACCGT \\
\hline 103 & TTTT TAAAGGTG GCAACATAGTAGAAAATACATACA \\
\hline 104 & TTTT GACGGGAG AATTAACTACAGGGAAGCGCATTA \\
\hline 105 & TTTT GCTTATCC GGTATTCTAAATCAGATATAGAAG \\
\hline 106 & TTTT CGACAAAA GGTAAAGTAGAGAATATAAAGTAC \\
\hline 107 & TTTT CGCGAGAA AACTTTTTATCGCAAGACAAAGAA \\
\hline 108 & TTTT ATTAATTA CATTTAACACATCAAGAAAACAAA \\
\hline 109 & TTTT TTCATCAA TATAATCCTATCAGATGATGGCAA \\
\hline 110 & TTTT AATCAATA TCTGGTCACAAATATCAAACCCTC \\
\hline 111 & TTTT ACCAGTAA TAAAAGGGATTCACCA GTCACACG TTTT \\
\hline 112 & CCGAAATCCGAAAATC CTGTTTGAAGCCGGAA \\
\hline 113 & CCAGCAGGGGCAAAAT CCCTTATAAAGCCGGC \\
\hline 114 & GCATAAAGTTCCACAC AACATACGAAGCGCCA \\
\hline 115 & GCTCACAATGTAAAGC CTGGGGTGGGTTTGCC \\
\hline 116 & TTCGCCATTGCCGGAA ACCAGGCATTAAATCA \\
\hline 117 & GCTTCTGGTCAGGCTG CGCAACTGTGTTATCC \\
\hline 118 & GTTAAAATTTTAACCA ATAGGAACCCGGCACC \\
\hline 119 & AGACAGTCATTCAAAA GGGTGAGAAGCTATAT \\
\hline 120 & AGGTAAAGAAATCACC ATCAATATAATATTTT \\
\hline 121 & TTTCATTTGGTCAATA ACCTGTTTATATCGCG \\
\hline 122 & TCGCAAATGGGGCGCG AGCTGAAATAATGTGT \\
\hline 123 & TTTTAATTGCCCGAAA GACTTCAAAACACTAT \\
\hline 124 & AAGAGGAACGAGCTTC AAAGCGAAGATACATT \\
\hline 125 & GGAATTACTCGTTTAC CAGACGACAAAAGATT \\
\hline 126 & GAATAAGGACGTAACA AAGCTGCTCTAAAACA \\
\hline 127 & CCAAATCACTTGCCCT GACGAGAACGCCAAAA \\
\hline 128 & CTCATCTTGAGGCAAA AGAATACAGTGAATTT \\
\hline 129 & AAACGAAATGACCCCC AGCGATTATTCATTAC \\
\hline 130 & CTTAAACATCAGCTTG CTTTCGAGCGTAACAC \\
\hline 131 & TCGGTTTAGCTTGATA CCGATAGTCCAACCTA \\
\hline 132 & TGAGTTTCGTCACCAG TACAAACTTAATTGTA \\
\hline 133 & CCCCGATTTAGAGCTT GACGGGGAAATCAAAA \\
\hline 134 & GAATAGCCGCAAGCGG TCCACGCTCCTAATGA \\
\hline 135 & GAGTTGCACGAGATAG GGTTGAGTAAGGGAGC \\
\hline 136 & GTGAGCTAGTTTCCTG TGTGAAATTTGGGAAG \\
\hline 137 & TCATAGCTACTCACAT TAATTGCGCCCTGAGA \\
\hline 138 & GGCGATCGCACTCCAG CCAGCTTTGCCATCAA \\
\hline 139 & GAAGATCGGTGCGGGC CTCTTCGCAATCATGG \\
\hline 140 & AAATAATTTTAAATTG TAAACGTTGATATTCA \\
\hline 141 & GCAAATATCGCGTCTG GCCTTCCTGGCCTCAG \\
\hline 142 & ACCGTTCTAAATGCAA TGCCTGAGAGGTGGCA \\
\hline 143 & TATATTTTAGCTGATA AATTAATGTTGTATAA \\
\hline 144 & TCAATTCTTTTAGTTT GACCATTACCAGACCG \\
\hline 145 & CGAGTAGAACTAATAG TAGTAGCAAACCCTCA \\
\hline
\end{tabular}




\begin{tabular}{|c|c|}
\hline 146 & GAAGCAAAAAAGCGGA TTGCATCAGATAAAAA \\
\hline 147 & TCAGAAGCCTCCAACA GGTCAGGATCTGCGAA \\
\hline 148 & CCAAAATATAATGCAG ATACATAAACACCAGA \\
\hline 149 & CATTCAACGCGAGAGG CTTTTGCATATTATAG \\
\hline 150 & ACGAGTAGTGACAAGA ACCGGATATACCAAGC \\
\hline 151 & AGTAATCTTAAATTGG GCTTGAGAGAATACCA \\
\hline 152 & GCGAAACATGCCACTA CGAAGGCATGCGCCGA \\
\hline 153 & ATACGTAAAAGTACAA CGGAGATTTCATCAAG \\
\hline 154 & CAATGACACTCCAAAA GGAGCCTTACAACGCC \\
\hline 155 & AAAAAAGGACAACCAT CGCCCACGCGGGTAAA \\
\hline 156 & TGTAGCATTCCACAGA CAGCCСTCATCTCCAA \\
\hline 157 & GTAAAGCACTAAATCG GAACCCTAGTTGTTCC \\
\hline 158 & AGTTTGGAGCCCTTCA CCGCCTGGTTGCGCTC \\
\hline 159 & AGCTGATTACAAGAGT CCACTATTGAGGTGCC \\
\hline 160 & ACTGCCCGCCGAGCTC GAATTCGTTATTACGC \\
\hline 161 & CCCGGGTACTTTCCAG TCGGGAAACGGGCAAC \\
\hline 162 & CAGCTGGCGGACGACG ACAGTATCGTAGCCAG \\
\hline 163 & GTTTGAGGGAAAGGGG GATGTGCTAGAGGATC \\
\hline 164 & СTTTCATCCCCAAAAA CAGGAAGACCGGAGAG \\
\hline 165 & AGAAAAGCAACATTAA ATGTGAGCATCTGCCA \\
\hline 166 & GGTAGCTAGGATAAAA ATTTTTAGTTAACATC \\
\hline 167 & CAACGCAATTTTTGAG AGATCTACTGATAATC \\
\hline 168 & CAATAAATACAGTTGA TTCCCAATTTAGAGAG \\
\hline 169 & TCCATATACATACAGG CAAGGCAACTTTATTT \\
\hline 170 & TACCTTTAAGGTCTTT ACCCTGACAAAGAAGT \\
\hline 171 & CAAAAATCATTGCTCC TTTTGATAAGTTTCAT \\
\hline 172 & TTTGCCAGATCAGTTG AGATTTAGTGGTTTAA \\
\hline 173 & AAAGATTCAGGGGGTA ATAGTAAACCATAAAT \\
\hline 174 & TTTCAACTATAGGCTG GCTGACCTTGTATCAT \\
\hline 175 & CCAGGCGCTTAATCAT TGTGAATTACAGGTAG \\
\hline 176 & CGCCTGATGGAAGTTT CCATTAAACATAACCG \\
\hline 177 & TTTCATGAAAATTGTG TCGAAATCTGTACAGA \\
\hline 178 & ATATATTCTTTTTTCA CGTTGAAAATAGTTAG \\
\hline 179 & AATAATAAGGTCGCTG AGGCTTGCAAAGACTT \\
\hline 180 & CGTAACGATCTAAAGT TTTGTCGTGAATTGCG \\
\hline 181 & ACCCAAATCAAGTTTT TTGGGGTCAAAGAACG \\
\hline 182 & TGGACTCCCTTTTCAC CAGTGAGACCTGTCGT \\
\hline 183 & TGGTTTTTAACGTCAA AGGGCGAAGAACCATC \\
\hline 184 & GCCAGCTGCCTGCAGG TCGACTCTGCAAGGCG \\
\hline 185 & CTTGCATGCATTAATG AATCGGCCCGCCAGGG \\
\hline 186 & ATTAAGTTCGCATCGT AACCGTGCGAGTAACA \\
\hline 187 & TAGATGGGGGGTAACG CCAGGGTTGTGCCAAG \\
\hline 188 & ACCCGTCGTCATATGT ACCCCGGTAAAGGCTA \\
\hline 189 & CATGTCAAGATTCTCC GTGGGAACCGTTGGTG \\
\hline
\end{tabular}




\begin{tabular}{|c|c|}
\hline 190 & TCAGGTCACTTTTGCG GGAGAAGCAGAATTAG \\
\hline 191 & CTGTAATATTGCCTGA GAGTCTGGAAAACTAG \\
\hline 192 & CAAAATTAAAGTACGG TGTCTGGAAGAGGTCA \\
\hline 193 & TGCAACTAAGCAATAA AGCCTCAGTTATGACC \\
\hline 194 & TTTTTGCGCAGAAAAC GAGAATGAATGTTTAG \\
\hline 195 & AAACAGTTGATGGCTT AGAGCTTATTTAAATA \\
\hline 196 & ACTGGATAACGGAACA ACATTATTACCTTATG \\
\hline 197 & ACGAACTAGCGTCCAA TACTGCGGAATGCTTT \\
\hline 198 & CGATTTTAGAGGACAG ATGAACGGCGCGACCT \\
\hline 199 & CTTTGAAAAGAACTGG CTCATTATTTAATAAA \\
\hline 200 & GCTCCATGAGAGGCTT TGAGGACTAGGGAGTT \\
\hline 201 & ACGGCTACTTACTTAG CCGGAACGCTGACCAA \\
\hline 202 & AAAGGCCGAAAGGAAC AACTAAAGCTTTCCAG \\
\hline 203 & GAGAATAGCTTTTGCG GGATCGTCGGGTAGCA \\
\hline 204 & ACGTTAGTAAATGAAT TTTCTGTAAGCGGAGT \\
\hline 205 & TTTT CGATGGCC CACTACGTAAACCGTC TATCAGGG \\
\hline 206 & TTTT CGGTTTGC GTATTGGGAACGCGCG GGGAGAGG \\
\hline 207 & TTTT TGTAAAAC GACGGCCATTCCCAGT CACGACGT \\
\hline 208 & TTTT GTAATGGG ATAGGTCAAAACGGCG GATTGACC \\
\hline 209 & TTTT GATGAACG GTAATCGTAGCAAACA AGAGAATC \\
\hline 210 & TTTT GGTTGTAC CAAAAACAAGCATAAA GCTAAATC \\
\hline 211 & TTTT CTGTAGCT CAACATGTATTGCTGA ATATAATG \\
\hline 212 & TTTT CATTGAAT CCСССTCAAATCGTCA TAAATATT \\
\hline 213 & TTTT GGAAGAAA AATCTACGACCAGTCA GGACGTTG \\
\hline 214 & TTTT TCATAAGG GAACCGAAAGGCGCAG ACGGTCAA \\
\hline 215 & TTTT GACAGCAT CGGAACGAACCCTCAG CAGCGAAA \\
\hline 216 & TTTT AACTTTCA ACAGTTTCTGGGATTT TGCTAAAC TTTT \\
\hline Loop1 & AACATCACTTGCCTGAGTAGAAGAACT \\
\hline Loop2 & TGTAGCAATACTTCTTTGATTAGTAAT \\
\hline Loop3 & AGTCTGTCCATCACGCAAATTAACCGT \\
\hline Loop4 & ATAATCAGTGAGGCCACCGAGTAAAAG \\
\hline Loop5 & ACGCCAGAATCCTGAGAAGTGTTTTT \\
\hline Loop6 & TTAAAGGGATTTTAGACAGGAACGGT \\
\hline Loop7 & AGAGCGGGAGCTAAACAGGAGGCCGA \\
\hline Loop8 & TATAACGTGCTTTCCTCGTTAGAATC \\
\hline Loop9 & GTACTATGGTTGCTTTGACGAGCACG \\
\hline -oop10 & GCGCTTAATGCGCCGCTACAGGGCGC \\
\hline
\end{tabular}


Sequences of the modified staple strands and the substrate \& DNAzyme strands:

\begin{tabular}{|c|c|}
\hline & CATTGTTT \\
\hline$L_{1}-104$ & $\begin{array}{l}\text { TTTGACGGGAGAATTAACTACAGGGAAGCGCATTATTTCATTC } \\
\text { TCATTGTTT }\end{array}$ \\
\hline $\mathrm{L}_{1}-107$ & GAAAACTTTTTATCGCAAGACAAAGAATTTCATTCT \\
\hline$L_{1}-110$ & $\begin{array}{l}\text { TTTTAATCAATATCTGGTCACAAATATCAAACCCT } \\
\text { TCATTGTTT }\end{array}$ \\
\hline $\mathrm{L}_{2}-206$ & 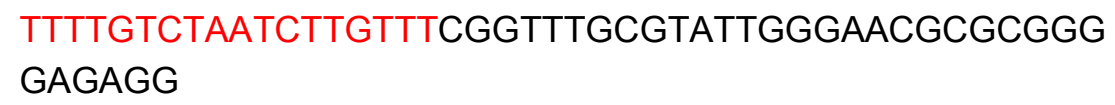 \\
\hline & t) \\
\hline $\mathrm{L}_{2}-212$ & $\begin{array}{l}\text { TTTTGTCTAATCTTGTTTCATTGAATCCCCCTCAAATCGTCATAA } \\
\text { ATATT }\end{array}$ \\
\hline $\mathrm{L}_{2}-215$ & $\begin{array}{l}\text { TTTTGTCTAATCTTGTTTGACAGCATCGGAACGAACCCTCAGCA } \\
\text { GCGAAA }\end{array}$ \\
\hline$S_{1}$ & ACTCACTATArGGAAGAGATGAAACAATG \\
\hline $\mathrm{C}_{1}$ & SATC \\
\hline & $\begin{array}{l}\text { GGCTCAGAACGAAAATAAATCCTCATTAAATGATATTC, } \\
\text { AA }\end{array}$ \\
\hline & $\begin{array}{l}\text { ATCAGAACGAAAGACGGGAGAATTAACTACAGGGAAGCG } \\
\text { A }\end{array}$ \\
\hline & CAGAACGAAACGCGAGAAAACTTTTTATCGCAAGACAA \\
\hline & GAACGAAAAATCAATATCTGGTCACAAATATCAAACC \\
\hline$L_{4}-206$ & $\begin{array}{l}\text { SGGTTTGCGTATTGGGAACGCGCGGGGAGAGGAAACTCGGAA } \\
\text { GTGA }\end{array}$ \\
\hline $\mathrm{L}_{4}-209$ & $\begin{array}{l}\text { GATGAACGGTAATCGTAGCAAACAAGAGAATCAAACTCGGAAC } \\
\text { GTGA }\end{array}$ \\
\hline $\mathrm{L}_{4}-212$ & АATTGAATCCCCCTCAAATCGTCATAAATATTAAACTCGGAACG \\
\hline $\mathrm{L}_{4}-215$ & $\begin{array}{l}\text { GACAGCATCGGAACGAACCCTCAGCAGCGAAAAAACTCGGAAC } \\
\text { GTGA }\end{array}$ \\
\hline $\mathrm{S}_{2}$ & $\begin{array}{l}\text { TTTCGTTCTGAGCCGCTGAGGACACrAGGAAGAGATGTTTCACG } \\
\text { TTCCGAGTTT }\end{array}$ \\
\hline & CATCTCTTAACGG \\
\hline & Biotin-TTTTCC \\
\hline & AAAAG IACAA CGG \\
\hline
\end{tabular}


